\title{
MUJERES Y NIÑAS INDÍGENAS DESAPARECIDAS Y ASESINADAS: Femicidio, Feminicidio, y Genocidio en el Contexto Canadiense ${ }^{1}$
}

\author{
MISSING AND MURDERED INDIGENOUS WOMEN AND GIRLS: \\ Femicide, Feminicide and Genocide in the Canadian Context
}

\section{MULHERES E MENINAS INDÍGENAS DESAPARECIDAS E ASSASSINADAS: Femicídio, Feminicídio e Genocídio no Contexto Canadense}

\section{Paulina García-Del Moral}

Professora Assistente, Departamento de Sociologia e Antropologia, Universidade de Guelph pgarciad@uoguelph.ca

\section{Resumen}

A pesar de enfatizar las semejanzas entre los feminicidios de Ciudad Juárez y las desapariciones y asesinatos de mujeres y niñas indígenas en Canadá (MMIWG por sus siglas en inglés), las activistas y académicas indígenas no utilizan el concepto de feminicidio para enmarcar este tipo de violencia. Las académicas usan el término de genocidio y las activistas transnacionales la enmarcan como discriminación interseccional y una violación a los derechos humanos. Ambas perspectivas se enfocan en la colonización como eje central.

Palabras clave: mujeres y niñas indígenas; genocidio; femicidio/ feminicidio.

\footnotetext{
${ }^{1}$ Este artículo se basa casi en su totalidad en trabajos académicos y documentos escritos en inglés. Todas las traducciones son de la autora.
} 


\begin{abstract}
Despite highlighting the parallels between feminicides in Ciudad Juarez and missing and murdered Indigenous women and girls (MMIWG) in Canada, Indigenous activists and scholars do not use the concept of feminicide to frame this violence. Indigenous feminist scholars use the term genocide, while, in their transnational advocacy, Indigenous activists frame this violence as intersectional discrimination and a human rights violation. These approaches make colonialism central to MMIWG.
\end{abstract}

Keywords: Indigenous women and girls; genocide; femicide/feminicide.

\title{
Resumo
}

Apesar de enfatizar as semelhanças entre os feminicídios de Ciudad Juárez e os desaparecimentos e assassinatos de mulheres e meninas indígenas no Canadá (MMIWG, por suas siglas em inglês), as ativistas e acadêmicas indígenas não usam o conceito de feminicídio para enquadrar este tipo de violência. As acadêmicas usam o termo genocídio e as ativistas transnacionais o enquadram como discriminação interseccional e uma violação dos direitos humanos. Ambas perspectivas enfocam o colonialismo como eixo central.

Palavras-chave: mulheres e meninas indígenas; genocídio; femicídio/feminicídio. 
egún las activistas indígenas de Canadá, existe una situación paralela notable entre la inacción del estado para prevenir, investigar y sancionar las desapariciones y los asesinatos de mujeres y niñas indígenas en Canadá y el contexto de impunidad que definen las desapariciones y los asesinatos de mujeres en Ciudad Juárez, México (ANDERSON, 2016; ANDERSON; KUBIK; HAMPTON，2010; NWAC; FAFIA; UM, 2012, p. 27; NWAC; FAFIA, 2013, p. 38) ${ }^{2}$. A pesar de ello, el Comité Nacional de Investigación sobre Mujeres y Niñas Indígenas Desaparecidas y Asesinadas (missing and murdered Indigenous women and girls, MMIWG) (BULLER et al., 2019, p. 5) caracterizó esta violencia como "genocidio basado en raza, identidad, y género" relacionado con prácticas e ideologías coloniales, en vez de caracterizarla como feminicidio, el término comúnmente usado para enmarcar la violencia contra las mujeres en Ciudad Juárez $^{3}$. Esta conceptualización contrasta con la mayoría de las definiciones del feminicidio, aún aquellas que proponen que se entienda como genocidio (e.g. LAGARDE, 2004), ya que no privilegian la misoginia o el patriarcado como causas de

\footnotetext{
${ }^{2}$ Mi uso del término "indígena" se refiere a los pueblos de las Primeras Naciones (First Nations), Métis, e Inuit de Canadá.

${ }^{3}$ El Comité Nacional de Investigación sobre Mujeres y Niñas Indígenas Desaparecidas y Asesinadas se creó en parte como respuesta del gobierno Liberal de Justin
}

esta violencia. Al contrario, ésta adopta una perspectiva interseccional que también considera la colonización. De hecho, las referencias a MMIWG como feminicidios o femicidios son escasas en la literatura académica canadiense, pero este no es el caso con el término de genocidio. Esto sugiere que las académicas y activistas indígenas no tienden a ver al feminicidio o femicidio como conceptos relevantes para capturar conceptualmente o atender las experiencias de violencia de las mujeres y niñas indígenas en el contexto canadiense, posiblemente apuntando a algunas limitaciones conceptuales de estos términos no sólo en Canadá, sino también en Latinoamérica y en otras partes del mundo.

Este artículo se basa en las publicaciones de la Asociación de Mujeres Nativas de Canadá (Native Women's Association, NWAC) y la Alianza Feminista Canadiense para Acción Internacional (Canadian Feminist Alliance for International Action, FAFIA) para analizar cómo las activistas indígenas han enmarcado el fenómeno de MMIWG en Canadá. Sitúo mi análisis en un resumen (no exhaustivo) sobre la violencia contra las mujeres y niñas indígenas en Canadá, así

Trudeau al activismo de mujeres indígenas y feministas, como se describirá en este artículo. Al fenómeno de mujeres y niñas indígenas desaparecidas y asesinadas se le conoce como MMIWG, o sea missing and murdered Indigenous women and girls. 
como una revisión de la literatura académica sobre este tema. El propósito de este artículo es contribuir al diálogo transnacional actual sobre la relación entre el colonialismo y las violencias extremas contra las mujeres indígenas y racializadas y el papel conceptual que el feminicidio podría jugar tanto para comprenderlas como para combatirlas (FIGUEROA ROMERO et al., 2017). El artículo no argumenta en contra de que se sigan usando los términos de feminicidio o femicidio en Latinoamérica o en otros países. Sin embargo, sugiere que el uso de estos conceptos debe de adoptar una perspectiva antirracista y que esté abierta a las críticas que puedan hacerle las mujeres indígenas con base en su propia interpretación de sus experiencias de violencia.

\section{Mujeres y Niñas Indígenas Desaparecidas y Asesinadas en Canadá (MMIWG)}

La violencia contra mujeres y niñas indígenas es un problema grave y extenso que sólo recientemente empezó a recibir atención gubernamental. Como resultado existe una falta de claridad sobre cuántas mujeres y niñas indígenas han desparecido o han sido asesinadas en las

\footnotetext{
${ }^{4}$ La Real Policía Montada de Canadá es el servicio policial nacional canadiense. En algunas provincias, también
}

últimas décadas. Las organizaciones indígenas de la sociedad civil, tales como NWAC, han estimado que hay al menos 663 mujeres y niñas desaparecidas o asesinadas entre 1986 y 2013 (NWAC; FAFIA, 2013). Además, NWAC estableció que 153 mujeres indígenas fueron asesinadas entre el 2000 y el 2013, representando el 10\% del número total de homicidios de mujeres en Canadá, aunque las mujeres indígenas representan apenas el 3\% de la población femenina (NWAC; FAFIA, 2013). En el 2014, la Real Policía Montada de Canadá (Royal Canadian Mounted Police, RCMP) reportó que hubo 1,107 homicidios de mujeres y niñas indígenas documentados por la policía entre 1980 y el 2012, y 164 desapariciones ${ }^{4}$. En ese informe, la RCMP (2014, p. 9) declaró que las mujeres indígenas representaban el $16 \%$ de todas las mujeres víctimas de homicidio. Información más reciente de Statistics Canada (2020) demuestra que 185 mujeres indígenas fueron asesinadas entre el 2014 y el 2018. Sobresalta que el informe final del Comité Nacional de Investigación sobre MMIWG (BULLER et al., 2019, p. 55) indique que las mujeres y niñas indígenas tienen una probabilidad 12 veces mayor de ser víctimas de homicidio o desaparición que mujeres que no son indígenas, siendo 16 veces mayor el riesgo si se les compara

tiene jurisdicción para ejercer como fuerza policial en municipios o ciudades. 
con mujeres blancas. Mientras tanto, datos del censo nacional han demostrado consistentemente que las tasas de abuso sexual, maltrato, y violencia conyugal son significativamente más altas para mujeres indígenas que para las mujeres no indígenas, y que las mujeres indígenas que sufren violencia conyugal tienden a temer por su vida con más frecuencia (BOYCE, 2016; BRZOZOWSKI; TAYLOR-BUTTS; JOHNSON，2006; DEPARTMENT OF JUSTICE CANADA, 2017). En suma, aunque haya una falta de claridad con respecto a la cifra precisa de mujeres y niñas indígenas desaparecidas y asesinadas queda claro que ellas tienen un mayor riesgo de ser víctimas de violencia tanto dentro como fuera de sus comunidades.

Sin embargo, tampoco está claro si los hombres indígenas son los principales autores de estos crímenes (INNES; ANDERSON, 2018). Datos de Statistics Canada demuestran que los hombres indígenas están sobrerrepresentados como personas acusadas de homicidio (BRZOZOWSKI; TAYLOR-BUTTS; JOHNSON, 2006, p. 8), mientras que el reporte de la RCMP (2014, p.12) indica que más del $90 \%$ de las víctimas conocían a sus asesinos. Este reporte también revela que la proporción de mujeres indígenas asesinadas por sus cónyuges (29\%) es menor que la de mujeres no indígenas (41\%). A su vez,
NWAC (2010, p. 29) encontró que las mujeres indígenas tienen una probabilidad tres veces mayor de ser asesinadas por extraños que mujeres que no son indígenas. A pesar de la falta de claridad de estas cifras, el anterior ministro de Asuntos Indígenas y Desarrollo del Norte de Canadá (Aboriginal Affairs and Northern Development Canada), Bernard Valcourt, aseguró que los hombres indígenas eran responsables de asesinar al 70\% de las mujeres indígenas identificadas en el reporte del 2014 de la RCMP (INNES; ANDERSON, 2018). La RCMP confirmó esta declaración poco después, enfatizando la relación entre estos crímenes y la violencia doméstica en la actualización de su reporte inicial en el 2015 (RCMP, 2015). Para Innes y Anderson (2018), si bien algunos de estos datos pudiesen sugerir que los homicidas son hombres indígenas, no es posible saberlo con seguridad porque "no hay un desglose por raza de los homicidas en las estadísticas oficiales." Por lo tanto, activistas indígenas también han sido muy críticas del uso selectivo de las estadísticas por parte de la RCMP con el propósito de culpar a los hombres indígenas por esta violencia y así "permitir que el gobierno escape de su responsabilidad por la discriminación a largo plazo en contra de los pueblos indígenas y también de su mediocre desempeño en la investigación de 
las desapariciones y los asesinatos de niñas y mujeres indígenas" (LSC, 2015, p. 9).

En efecto, desde que Amnistía Internacional (2004) publicó Stolen Sisters (Hermanas Robadas) para llamar la atención nacional e internacionalmente sobre la situación de violencia contra las mujeres y niñas indígenas, activistas, organizaciones de la sociedad civil, y académicas han argumentado insistentemente que la historia colonial de Canadá y su legado de racismo y sexismo sistémicos son la raíz de la violencia que afecta tan desproporcionadamente a las mujeres y niñas indígenas, robándoles la vida y sus derechos (ANDERSON; CAMPBELL; BELCOURT, 2018; FEINSTEIN; PEARCE, 2015; GREEN, 2017; HARGREAVES, 2017; LAVELLHARVARD; BRANT, 2016; PALMATER, 2016; SMITH, 2005). Este legado se ve reflejado en la respuesta institucional, ya sea ausente, deficiente, o inconsistente, hacia esta epidemia de violencia al nivel provincial y federal. Por ejemplo, las activistas indígenas han documentado que la policía frecuentemente se basa en estereotipos racistas y sexistas para descartar reportes de desaparición de mujeres y niñas indígenas, especialmente si son trabajadoras sexuales o se percibe que llevan un "estilo de vida de alto riesgo" (CULHANE, 2003; EBERTS, 2017;
GOULDING，2001; HARGREAVES, 2017; LAVELL-HARVARD; BRANT, 2016; NWAC, 2010; NWAC; FAFIA, 2013). Las omisiones de la Policía de Vancouver, así como la confusión sobre la jurisdicción de la RCMP, permitió que el asesino serial Robert Pickton se aprovechara de sexoservidoras, muchas de ellas indígenas, que trabajaban en la marginada zona de Downtown Eastside en el centro este de Vancouver (CULHANE, 2003; HARGREAVES, 2017; O'REILLY; FLEMING, 2016, p. 59). El ADN de 33 mujeres fue encontrado en la granja de cerdos de Pickton, quien fue acusado y sentenciado por el homicidio en segundo grado de seis mujeres, Sereena Abotsway, Mona Wilson, Andrea Joesbury, Georgina Papin, Marnie Frey, y Brenda Wolfe (O’REILLY; FLEMING, 2016).

La policía también actuó con lentitud para investigar los casos de desapariciones y asesinatos de mujeres $\mathrm{y}$ niñas indígenas de la Autopista de las Lágrimas (Highway of Tears), un tramo de $724 \mathrm{~km}$ de la Autopista 16 entre las poblaciones de Prince Rupert y Prince George, en Columbia Británica. Fue tan sólo en el 2002, cuando desapareció Nicole Hoar, una joven blanca, que la policía comenzó a actuar (O’REILLY; FLEMING, 2016, p. 53). Bajo el Proyecto E-PANA, creado en el 2005, la RCMP está 
investigando 18 casos, los cuales incluyen también incidentes que sucedieron en autopistas cercanas, aunque las activistas indígenas han reclamado que muchas más mujeres y niñas han sido secuestradas o asesinadas (SABO, 2016). De los 18 casos de E-PANA, 13 son homicidios que en su mayoría siguen sin ser resueltos $(\mathrm{SABO}$, 2016). Asimismo, muchas mujeres y niñas indígenas han sido desaparecidas $\mathrm{o}$ asesinadas al este de Canadá; muchos de estos casos siguen sin ser resueltos o han recibido poca atención (SAVARESE, 2016). La discriminación racial es predominante aquí también, como lo evidencia el trato diferenciado que recibió la familia de Loretta Saunders por parte de la policía cuando se pensó inicialmente que era una mujer blanca. Saunders era una mujer Inuk de 26 años de Newfoundland y Labrador, asesinada en Halifax, Nueva Escocia, en 2014. Aunque sus asesinos fueron capturados y llevados a juicio, el predominio de la discriminación racial incitó la creación de la Coalición de Estrategia Legal sobre Violencia contra las Mujeres Indígenas (Legal Strategy Coalition on Violence against Indigenous Women, LSC) a través del Fondo de Acción y Educación Legal de Mujeres (Women's Legal Action and Education Fund, LEAF).

Ciertamente, para las organizaciones indígenas de la sociedad civil, los casos sin resolver no son una excepción; al contrario, han indicado que la mayoría de los asesinatos de mujeres y niñas indígenas siguen sin resolverse y en el $40 \%$ de los casos no se han presentado cargos en contra de sus autores (NWAC, 2010). Estas acusaciones ponen en duda las figuras de la RCMP, las cuales sugieren que la tasa de resolución de estos asesinatos es del $80 \%$ al $100 \%$ a lo largo del país, comparable con la de mujeres no indígenas (LSC, 2015). No obstante, la misma RCMP (2014, p. 14) admitió que los homicidios de mujeres indígenas involucradas en la industria del sexo $\mathrm{u}$ otras actividades ilegales tenía una tasa de resolución menor, del 60 al 65\%, aparentemente también comparable a la de otras mujeres que están igualmente involucradas en esas actividades. Sin embargo, un problema con estas figuras es que la RCMP no provee información sobre la proporción de casos que han resultado en cargos presentados y/o sentencias (DAWSON, 2016). No es sorprendente, por lo tanto, que haya discrepancias entre los datos de las activistas indígenas y la RCMP.

De acuerdo con Dawson (2016, p. 998), "la continua falta de datos sistemáticos sobre las cortes canadienses que permitan que los investigadores establezcan una relación entre las características de los casos y sus castigos ha 
obstaculizado la evaluación sistemática del sistema judicial y su respuesta a la violencia contra mujeres indígenas." Pese a ello, hay evidencia que sugiere que el sexismo y racismo institucional obstruyen severamente el acceso a la justicia de las mujeres indígenas. Por ejemplo, tuvieron que pasar 16 años para que tres de los cuatro asesinos de Helen Betty Osborne, una mujer Cree asesinada en 1971 en The Pas, Manitoba fueran llevados a juicio; sólo se le condenó a uno de ellos, otro fue exonerado, al tercero se le ofreció inmunidad a cambio de su testimonio, mientras que al cuarto nunca se le imputaron cargos (JACOBS, 2018). Los juicios de Pamela George y Cindy Gladue constituyen otro ejemplo. Pamela George era una mujer Salteux (Ojibway) que se prostituía ocasionalmente para sostener a su familia. En 1995, dos atletas blancos de la Universidad de Regina, Steven Kummerfield y Alex Ternowetsky, la mataron a golpes. Le tomó a la RCMP más de un mes arrestar a estos hombres porque enfocó su búsqueda en hombres indígenas o indigentes como los principales sospechosos del crimen (RAZACK, 2002, p. 125). Durante el juicio, el juez le dijo varias veces al jurado que "tuvieran en mente que la occisa era una prostituta," mientras que la defensa argumentó que "los chicos habían hecho cosas muy estúpidas, pero no habían cometido ningún crimen" (RAZACK, 2002, p. 125). Se estableció más tarde que, de hecho, los dos hombres les presumieron a sus amigos haber golpeado a "una india puta" antes de que fueran arrestados (JACOBS, 2018, p. 20; RAZACK, 2002, p. 139). Se les encontró culpable de homicidio involuntario, aunque no cumplieron sus sentencias de seis años.

En el 2011, Bradley Barton, un camionero blanco, mató a Cindy Gladue, una mujer Cree, en un hotel en Edmonton, Alberta. Gladue se desangró en la tina después de que Barton insertara su puño, y probablemente también un cuchillo, en su vagina, causando una herida de $11 \mathrm{~cm}$. La defensa argumentó que esto había sucedido durante "sexo rudo" al cual Gladue había accedido y por el cual Barton le había pagado (RAZACK, 2016, p. 286); esto a pesar de que los niveles de alcohol en la sangre de Gladue eran demasiado altos como para que ella hubiera podido dar su consentimiento (FIDAN; DOEMEZ, 2016, p. 117). Al igual que en el juicio de Pamela George, la defensa enfatizó que Gladue era sexoservidora. Además, tanto la defensa como la Corona (el fiscal) se refirieron continuamente a ella como "la mujer nativa" en vez de por su nombre. Pero este fue el menor de los insultos al cuerpo y a la memoria de Gladue: su vagina fue expuesta como evidencia del crimen durante el juicio para sustentar las acusaciones de que Barton había utilizado un cuchillo (RAZACK, 
2016). Barton fue exonerado al final, aunque el caso fue apelado y habrá otro juicio. Lo que demuestran estos ejemplos es el absoluto desdeño con el cual se le trata a las mujeres indígenas, especialmente si son trabajadoras sexuales, en el sistema judicial canadiense.

El exonerar o dar sentencias poco severas a los asesinos no es la única forma en que el sistema judicial le falla a las mujeres indígenas. En el 2013, Human Rights Watch publicó un reporte que documenta los abusos de David Ramsay, un juez en la provincia de Columbia Británica. Ramsay abusó repetidamente de niñas indígenas de 12 a 17 años durante los nueve años que mantuvo su cargo (1991-2000). Ramsay seleccionaba a sus víctimas entre las jóvenes que aparecían ante él en la corte, pero a veces las recogía en la calle. Aunque se le declaró culpable y fue condenado en el 2004, Human Rights Watch (2013) y activistas indígenas argumentan que la complicidad de oficiales de la RCMP en esta violencia sigue impune. De hecho, Human Rights Watch (2013) y varias activistas han hecho acusaciones en contra de agentes de la RCMP por abusar de niñas y mujeres indígenas rutinariamente con impunidad (LINDBERG; CAMPEAU; CAMPBELL, 2012; PALMATER, 2016). También hay evidencia de que policías en Quebec han cometido hechos similares; pero a pesar de ello, se les ha suspendido de su cargo con paga, su sindicato les ha defendido, y ni el gobierno de federal o de Quebec han condenado sus acciones (EBERTS, 2017, p. 74; PALMATER, 2016, p. 274).

Otras instituciones canadienses cuyo deber es proteger a las más personas vulnerables también han fallado a las niñas indígenas. Según NWAC (2010), muchas de las mujeres y niñas asesinadas habían estado a cargo de agencias para el bienestar de menores. Esta situación se puede ejemplificar con el trágico caso de Tina Fontaine, una niña Sagkeeng de 15 años. El 17 de agosto de 2014, el cuerpo de Fontaine, envuelto en plástico y una colcha, fue descubierto en el Río Rojo de Winnipeg, Manitoba. Por gran parte de su vida, Fontaine vivió con su abuela materna, aunque en ocasiones estuvo a cargo del Servicio para Niños y Familias (CFS por sus siglas en inglés) de Manitoba, ya que su madre había perdido su custodia (CONN, 2019; MACY, 2019). La madre de Fontaine sufrió de abuso sexual durante su infancia mientras estaba a cargo de CFS, aunque la agencia parece no haber intervenido para protegerla (MACY, 2019, p. 16). El padre de Fontaine fue asesinado a golpes cuando ella tenía 12 años, pero no se le ofreció ningún apoyo psicológico (CONN, 2019; MACY, 2019). 
Durante una visita a su madre en el verano de 2014, Fontaine fue asesinada a pesar de estar bajo el cargo del CFS. Después de requerir el auxilio de la policía de Winnipeg porque un hombre la jalaba del brazo en la calle (MACY, 2019, p. 38), Fontaine fue alojada en un hotel por el CF토, aunque luego abandonó el alojamiento. Posteriormente estuvo en un albergue para jóvenes vulnerables hasta que fue reportada como desaparecida el 31 de julio de ese mismo año. No obstante este reporte, la policía de Winnipeg la dejó ir a pesar de haberla visto en un coche en compañía de un hombre mayor durante una detención de tráfico- (MACY, 2019). Poco después de este incidente, Fontaine fue encontrada inconsciente en un callejón y admitida en un hospital, en donde le dijo a una trabajadora del CFS que había estado frecuentando a Raymond Cormier, un hombre de 53 años. El CFS la alojó de nueva cuenta en un hotel, pero Fontaine volvió a irse. La última vez que se le vio con vida fue el nueve de agosto (CONN, 2019). Cormier, acusado de su muerte, fue exonerado del crimen. El caso no fue apelado.

Es evidente que el Estado canadiense ha estado consciente del alto riesgo de victimización que encaran las mujeres y niñas indígenas, al igual que de las severas deficiencias del sistema judicial y de otras instituciones. Sin embargo, ha escogido no actuar para atender estos problemas o, peor aún, en ocasiones ha obstruido los esfuerzos de las activistas indígenas por documentarlos (KUBIK; BOURSASSA，2016). La LSC (2016) revisó 58 reportes acerca de la violencia y discriminación en contra de mujeres y niñas indígenas publicados entre 1994 y 2015 que contienen más de 700 recomendaciones, pocas de las cuales han sido implementadas. Además, el Comité Nacional de Investigación sobre MMIWG ha tenido precursores, incluyendo la Comisión de Investigación Indígena de la Justicia de Manitoba en 1988 y la Comisión para Investigar las Desapariciones de Mujeres de la Columbia Británica en el año 2010 (KUBIK; BOURASSA, 2016). Estas comisiones concluyeron que las mujeres indígenas son un blanco de asesinos y que existe una relación entre su marginación social y la vulnerabilidad individual a la violencia de cada víctima. A su vez, las comisiones enfatizaron, tal como las activistas indígenas, el impacto de las relaciones coloniales que persisten en el presente entre Canadá y los pueblos indígenas. La Comisión de la Verdad y Reconciliación (2008-2015) también apoyó estas conclusiones, aunque no se enfocó exclusivamente en la violencia contra mujeres y niñas indígenas. Así lo hicieron igualmente los reportes de la Comisión 
Interamericana de Derechos Humanos (CIDH) (2014) y el Comité para la Eliminación de la Discriminación contra las Mujeres (Comité CEDAW) de la Organización de las Naciones Unidas (ONU) después de sus visitas a Canadá para investigar el problema de MMIWG.

Aunque la inacción ha sido probablemente el modus operandi más común de los gobiernos canadienses para con la epidemia de MMIWG, el gobierno conservador de Stephen Harper (20062015) se caracterizó por su profunda resistencia para reconocer la seriedad del problema. Harper declaró en una entrevista posterior a la publicación del informe de la RCMP que las desapariciones y/o asesinatos de más de 1000 mujeres y niñas indígenas "no estaban en su radar" (LAVELL-HARVARD; BRANT, 2016, p. 3). Después del asesinato de Tina Fontaine, Harper propuso en otra entrevista que este problema no debería de verse como un "fenómeno sociológico" (KUBIK; BOURASSA, 2016, p. 34). Su gobierno se rehusó repetidamente a crear un comité nacional para investigar esta violencia. El Comité Nacional de Investigación sobre MMIWG fue creado sólo después de que el candidato liberal, Justin Trudeau, derrotara a Harper en las elecciones federales del 2015. Trudeau se había comprometido a crear el Comité Nacional de Investigación durante su campaña; sin embargo, éste ha sido ampliamente criticado (NWAC, 2016, 2017) y sus 231 recomendaciones o "llamadas de justicia" no han sido implementadas. La inacción del Estado y la falta de responsabilidad de sus diferentes instituciones han contribuido así al problema de MMIWG.

\section{Femicidio, Feminicidio, y Genocidio en el Contexto Canadiense}

Las referencias a los asesinatos de mujeres y niñas indígenas como femicidio (femicide) en la literatura académica canadiense son poco comunes. El término femicidio "ganó relevancia" en Canadá después de que Mark Lépine asesinara a 14 mujeres que estudiaban ingeniería en el Ecóle Polytechnique de Montreal en 1989, acusándolas de ser feministas y de haber tomado su lugar en ese programa (DAWSON, 2018). Históricamente, la mayoría de los estudios sobre el femicidio en Canadá se han enfocado en el homicidio de mujeres en el contexto de la violencia íntima, ya sea el homicidio de pareja íntima o el uxoricidio (CRAWFORD; GARTNER; DAWSON, 1998; DAWSON, 2016; DAWSON; GARTNER, 1998). En estos estudios, el femicidio íntimo se entiende como el resultado de la suposición de 
propiedad sobre las mujeres por parte de los hombres. Esta definición refleja los orígenes de este concepto en el feminismo radical, originalmente definido como el asesinato misógino de mujeres por hombres (RADFORD; RUSSELL, 1992; RUSSELL; HARMES, 2002).

Aunque esta literatura discute la sobrerrepresentación de las mujeres indígenas como víctimas de femicidio íntimo (DAWSON; GARTNER, 1998; DAWSON, 2016), estas experiencias no fueron el principal foco de análisis. Más recientemente, la socióloga feminista Myrna Dawson, líder en el estudio del femicidio en Canadá, ha hecho una llamada para que se use este término para referirse a los asesinatos de mujeres y niñas indígenas en general, como también lo ha hecho Brenda Anderson (2016). Dawson fundó el Observatorio Canadiense del Feminicidio y para la Justicia y Rendición de Cuentas (Canadian Femicide Observatory for Justice and Accountability, CFOJA) en la Universidad de Guelph. El CFOJA (DAWSON, 2018, p. 2) define el femicidio como "el asesinato de mujeres y niñas por el hecho de serlo a mano principalmente, pero no exclusivamente, de hombres." Según el sitio web del CFOJA (2020), “se usa el término femicidio para describir estos asesinatos porque el género sigue siendo un factor determinante en el riesgo a la violencia que enfrentan las mujeres y niñas indígenas en algunos contextos en comparación con los hombres indígenas.” A su vez, el CFOJA declara que "dado el rol de la violencia racializada y la impunidad documentada en los casos de asesinato de mujeres indígenas, el término de feminicidio que se ha popularizado en Latinoamérica también puede ser usado, ya que apunta a la impunidad de los asesinos, así como la respuesta deficiente del Estado hacia los crímenes." Sin embargo, el CFOJA no emplea el término feminicidio, $\mathrm{y}$ tampoco lo hace Brenda Anderson (2016), quien ha estudiado las semejanzas entre los asesinatos de mujeres en México y en Guatemala y los homicidios de mujeres y niñas indígenas en Canadá.

En efecto, aunque común en Latinoamérica, especialmente en México, el término feminicidio es casi desconocido en Canadá. Como lo explican Marcela Lagarde (2004) y Julia Monárrez (2002), las feministas mexicanas que acuñaron el concepto, feminicidio es la traducción al español de "femicide" usado en la literatura del feminismo radical (RADFORD; RUSSELL, 1992; RUSSELL; HARMES, 2002). Aunque existen varias definiciones de feminicidio, parece existir un consenso de que constituye un "crimen de Estado" porque el Estado es cómplice en la violencia al crear un contexto en el cual las mujeres y 
niñas pueden ser asesinadas con impunidad (FREGOSO; BEJARANO, 2010). Por eso Lagarde (2004) define el feminicidio como "genocidio misógino contra las mujeres" y también como un "crimen de lesa humanidad." Es sólo recientemente que el concepto de feminicidio se ha traducido al inglés como "feminicide" (FREGOSO; BEJARANO, 2010; MENJÍVAR; WALSH, 2016, 2017). A la fecha, sólo una autora ha usado el concepto de feminicidio en Canadá específicamente para referirse a los asesinatos de mujeres y niñas indígenas, argumentando a la vez que este concepto se debe de repensar desde una perspectiva decolonial para sí “considerar al género como una categoría de análisis necesaria, pero no definitiva" de la violencia (GARCÍA-DEL MORAL, 2018, p. 949).

En resumen, femicidio es el concepto original en inglés y ha sido dominante en el contexto canadiense. Es probable que su predominio se deba en parte también a barreras lingüísticas, pero también a la noción de que puede tener el mismo énfasis en la impunidad e inacción del estado que caracterizan a las definiciones de feminicidio (DAWSON, 2018).

Las mujeres indígenas, ya sea como académicas y/o activistas, han producido la mayoría de la literatura sobre MMIWG (véase e.g. GREEN, 2017; HARGREAVES, 2017; KOUKKANEN,
2014; LAVELL-HARVARD; BRANT, 2016; LUCCHESI, 2019; MILLION, 2011; PALMATER, 2016; SMITH, 2005), frecuentemente en conversación con el trabajo de feministas antirracistas (e.g. JIWANI; YOUNG, 2006; RAZACK 2002, 2016). Aunque el feminismo es un concepto muy debatido entre las mujeres indígenas, muchas de estas autoras han adoptado una perspectiva feminista indígena que se enfoca en el "colonialismo como la condición estructural más urgente que afecta a las mujeres indígenas" (GREEN, 2017, p. 5; ARVIN; TUCK; MORRILL, 2013). De acuerdo con Green (2017, p. 45), es esta atención al colonialismo que distingue al feminismo indígena de otros feminismos, así como la incorporación de elementos centrales de culturas indígenas, incluyendo "la conexión casi universal con la tierra y el territorio a través de relaciones que se entienden como una responsabilidad sagrada predicada en reciprocidad y que definen la cultura y la identidad." Desde esta perspectiva, la violencia contra las mujeres y niñas indígenas "no es simplemente una herramienta de control patriarcal, sino que funciona como una herramienta del racismo y del colonialismo," como lo ha dicho Andrea Smith (2005, p. 1). Es en esta literatura que ha emergido la noción de que la violencia contra las mujeres y niñas indígenas amonta a un genocidio. 
Para sostener este argumento, las académicas feministas indígenas han desarrollado una "perspectiva teórica del colonialismo de colonos o de asentamiento (settler colonialism) como un sistema dominante de opresión que históricamente ha organizado y continúa organizado la vida en la sociedad canadiense" (BOURGEOIS, 2018). El colonialismo de asentamiento es una forma distinta de colonización, cuyo objetivo principal es adquirir tierra para asentarse y obtener control sobre sus recursos, en lugar de la extracción de recursos para la metropole (GLENN, 2015, p. 54). Para llevar a cabo este objetivo en lo que ahora es Canadá se volvió imperativo para los colonizadores británicos y franceses eliminar a los ocupantes originales de la tierra y después asegurar el territorio (GLENN, 2015, p. 54; STASIULIS; JHAPPAN, 1995). Por una parte, estos violentos procesos fueron de la mano del asesinato masivo de pueblos indígenas, de su traslado forzado de sus territorios para abrir camino al asentamiento de los colonos blancos, de su confinamiento a reservas a los márgenes de estos asentamientos $\mathrm{y}$, finalmente, de la adopción de políticas de asimilación (GLENN, 2015, p. 54). Por otra parte, estos procesos requirieron de la imposición de nociones modernistas de la tierra y sus recursos como propiedad que contrastaban con la visión cosmológica de los pueblos indígenas en cuanto a su relación con la tierra (GLENN, 2015, p. 54). De hecho, la ausencia de tal régimen de propiedad llevó a los pobladores europeos a adoptar el mito de terra nullius o vacuum domicilium, o sea, del territorio vacío, para así legitimar su reclamo a la tierra (SMITH, 2005; STASIULIS; JHAPPAN, 1995).

A este mito le acompañó la construcción de una jerarquía racial que definió a los pueblos indígenas no sólo como seres primitivos, sino como sub humanos y por lo tanto fundamentalmente inferiores a los pobladores blancos (BOURGEOIS, 2017). También conllevó la marginación política y socioeconómica de comunidades indígenas (BOURGEOIS, 2017, 2018; RIEL-JOHNS, 2016; RAZACK, 2002). El resultado de este proyecto de asentamiento colonial fue, como lo ha dicho la académica feminista antirracista Evelyn Nakano Glenn (2015, p. 58) "una identidad racializada y de género que normalizó la masculinidad blanca.”

La violencia del colonialismo de asentamiento, la ideología que usó para legitimar esa nueva identidad nacional, y sus efectos raciales/racistas y de género "plantaron las semillas" para la epidemia actual de MMIWG (GREEN, 2017; LAVELL-HARVARD; BRANT， 2016; RAZACK, 2002; SMITH, 2005). Como lo han dicho las feministas indígenas, la 
violencia sexual contra las mujeres y niñas indígenas ha ido de la mano del robo, e incluso de la violación, de las tierras indígenas (BOURGEOIS, 2017, 2018; MILLION, 2011; SMITH, 2005). Parte integral de esa violencia también fue el desmantelamiento de la organización matrilineal de las sociedades indígenas, así como el ataque al poder de las mujeres indígenas en ellas (JACOBS, 2018; LAVELL-HARVARD; BRANT， 2016; RIEL-JOHNS, 2016). Esto fue necesario para concretar el dominio heteropatriarcal sobre el nuevo territorio como propiedad, así como sobre la organización doméstica en la forma de la familia nuclear "como el modelo de la organización del estado y sus instituciones" (ARVIN; TUCK, MORRIL, 2013, p. 13; EBERTS, 2017). La institucionalización del heteropatriarcado a través de la imposición religiosa y cultural de valores cristianos seguido de su codificación legal en el Indian Act (Acta India) jugaron un papel central (EBERTS, 2017; JACOBS, 2018).

Según Smith (2005, p. 23), el [hetero]patriarcado ha servido para "naturalizar" las jerarquías coloniales e “inscribir la dominación sobre el cuerpo de los colonizados." Para los pobladores blancos, la transgresión de los valores heteropatriarcales europeos de feminidad por parte de las mujeres indígenas por virtud de su posición de poder en sus sociedades y el ejercicio independiente de su sexualidad constituían un símbolo del "salvajismo indígena" y, por lo tanto, una llamada a "civilizarlas" (STEVENSON, 1999, p. 58). En particular, los misionarios cristianos representaron a las mujeres indígenas como "indias esclavizadas" (squaw drudges) que eran a la vez "promiscuas y moralmente corruptas"; por lo tanto, la religión cristiana las "liberaría de siglos de opresión" (ACOOSE, 1995; CARTER, 1997, p. 162). En vez de liberarlas, el discurso de la "india promiscua" justificó la superioridad de las mujeres blancas, cuya moralidad y pureza debían ser protegidas, y la inferioridad de las mujeres indígenas, convirtiéndolas así en "cuerpos inherentemente violables" (ACOOSE, 1995; RAZACK, 2002; SMITH, 2005, p. 10).

Más específicamente, el discurso de la "india promiscua" creó la imagen de las mujeres indígenas como prostitutas $\mathrm{y}$ legitimó la violencia sexual en su contra, así como la inacción por parte de las autoridades para castigarla (CARTER, 1997, p. 187; ERICKSON, 2011, p. 45). Para Razack (2002, 2016), este discurso de la otredad ha permitido que pobladores blancos se vean a sí mismos hasta hoy en día como sujetos que tienen derecho tanto a la tierra como a las mujeres indígenas. No 
sólo eso, las nociones raciales y generizadas de moralidad que forman parte de este discurso han moldeado el trato que se les da a las trabajadoras sexuales indígenas en el sistema judicial. El Código Penal de 1892 categorizó la "prostitución indígena" como una ofensa a la moralidad y, por lo tanto, como un delito procesable (ERICKSON, 2011). A diferencia de ello, la "prostitución no indígena" constituía un delito menor bajo la rúbrica de la disrupción del orden público (ERICKSON, 2011, p. 62). Al hacerlo, el Código Penal reforzó la legitimidad de las barreras raciales entre las reservas indígenas y los nuevos asentamientos blancos (CARTER, 1997; ERICKSON, 2011), mientras que facilitó la mayor criminalización de las mujeres indígenas que continúa hasta hoy día (SANGSTER citada in BOURGEOIS, 2018).

Desde un principio se incorporaron también las nociones heteropatriarcales de familia $\mathrm{y}$ propiedad al Indian Act, promulgada en 1876. Esta acta no sólo gobierna la relación entre los pueblos indígenas y el gobierno de Canadá, sino que determina quién tiene "estatus legal de indio" (Indian status) y es merecedor de los beneficios de las obligaciones de varios convenios (BOURGEOIS, 2018). El Indian Act privó a las mujeres indígenas de derechos civiles y políticos al prohibirles que votasen en elecciones de bandas y que ocuparan cargos públicos (BOURGEOIS, 2015; RIEL-JOHNS, 2016). También autorizó la discriminación de género bajo la sección 12(1)(b), posiblemente con objetivos de asimilación (JACOBS, 2018; SAVARESE, 2016). Por ejemplo, el matrimonio entre mujeres indígenas $\mathrm{y}$ hombres que no lo fueran resultaba en la pérdida de su estatus legal como-de indio y la capacidad correspondiente de pasar tanto ese estatus a sus hijos, así como el derecho de vivir en reservas. Los hombres indígenas no estaban sujetos a esta provisión. Como resultado fue posible excluir a las mujeres indígenas y a sus descendientes de sus comunidades y privarlas de su derecho a recursos gubernamentales.

Las mujeres indígenas han resistido esta provisión discriminatoria del Indian Act, disputándola primero en la década de 1970 ante cortes canadienses en los casos $A G$ v. Lavell y R. v. Bédard y, en 1985, ante el Comité de Derechos Humanos de la ONU en Lovelace v. Canada (EBERTS, 2017; NAGY, 2016). Lovelace v. Canada mandó que el gobierno canadiense reformara el Indian Act y reinstituyera el "estatus de indio" a las mujeres que lo habían perdido antes de 1985, aunque esta reforma se hizo de tal forma que siguió permitiendo la discriminación de género (EBERTS, 2017). La reforma se llevó a cabo a través del 
Proyecto de Ley C-31 que instituyó un "punto de corte" que seguía restringiendo el derecho de la segunda generación de descendientes de estas mujeres para reclamar su estatus (EBERTS, 2017; NAGY, 2016). Esta provisión del Indian Act fue disputada una vez más ante el Comité de Derechos Humanos en McIvor v. Canada, a pesar de críticas por parte de hombres líderes indígenas. Como lo había hecho previamente, el Comité de Derechos Humanos decidió en enero de 2019 que Canadá continuaba discriminando a las mujeres indígenas (DEER, 2019). En otras palabras, tanto la provisión discriminatoria en base al género del Indian Act como la negativa del gobierno canadiense para remediarla demuestran "la amenaza simbólica y física" que las mujeres indígenas y su capacidad de reproducir a la nación indígena siempre han significado para el orden colonial (LAVELLHARVARD; BRANT, 2016, p. 3).

Para las feministas indígenas, el Indian Act ha tenido implicaciones genocidas porque ha tratado de eliminar as las mujeres indígenas de manera simbólica al haber reducido el número de la población con "estatus de indio" y de esta forma ha permitido el robo continuo de tierras con el motivo de formar asentamientos y también proyectos de extracción, (KNOTT, 2018; LAVELL-HARVARD; BRANT, 2016, p.
4; RAY, 2018/2019; SMITH, 2005). La pérdida de este estatus también ha tenido consecuencias genocidas porque ha puesto a múltiples generaciones de mujeres indígenas en una condición socioeconómica precaria. Esta pobreza y la precariedad económica han mermado su capacidad de ejercer una maternidad plena, exacerbando así el trauma intergeneracional causado por las escuelas residenciales (LAVELLHARVARD; BRANT, 2016). Por más de un siglo, fue obligatorio que los niños y las niñas indígenas atendieran internados en donde tenían prohibido practicar su idioma y costumbres tradicionales $\mathrm{y}$, en muchas ocasiones, fueron sujetos a violencia física, sexual y psicológica de manera rutinaria. Estos internados o escuelas residenciales destruyeron la integridad de las comunidades indígenas en todo Canadá (LAVELL-HARVARD; BRANT, 2016). Los internados y el trauma intergeneracional resultante interrumpieron la capacidad de las mujeres indígenas de transmitirle a sus descendientes su cultura, idiomas, y costumbres. A su vez, el trauma intergeneracional está relacionado con la normalización de la violencia sexual entre las mujeres y niñas indígenas (KNOTT, 2018). Por otra parte, la falta de acceso legal a recursos ha llevado a muchas mujeres indígenas a recurrir al trabajo sexual de subsistencia, contribuyendo así a la trata de personas, la cual está ligada al problema de 
MMIWG

(BOURGEOIS,

2015;

LUCCHESI, 2019). Según las feministas indígenas, la trata o prostitución de mujeres indígenas en el contexto de la industria de extracción también refleja el deterioro de la relación ancestral entre las mujeres indígenas y la tierra como consecuencia del Indian Act y sus objetivos genocidas. En este contexto, tanto la tierra como las mujeres se vuelven "desechables" (KNOTT, 2018; RAY, 2018/2019). Es en este sentido que, de acuerdo con Ray (2018/2019, p. 114), "la prostitución, la industria petrolera, y la construcción de oleoductos fungen como sitios que sostienen las prácticas de dominación colonial."

En resumen, la violencia racial y de género que dio pie al nacimiento del estado canadiense continúa prosperando en la epidemia de MMIWG.

\section{Metodología y Data}

Para examinar la forma en que las activistas indígenas han enmarcado la violencia contra las mujeres y niñas indígenas me apoyo en la literatura sobre movimientos sociales como mi marco analítico. Esta literatura ha demostrado de manera convincente que los marcos de los movimientos sociales son importantes para determinar sus resultados, incluyendo el impacto de movilización que pueden tener, al igual que su capacidad de efectuar cambios sociales, políticos y legales (BENFORD; $\quad$ SNOW, 2000; MCCAMMON et al., 2001; MCCAMMON et al., 2007; PEDRIANA, 2006). Como "paquetes de interpretación de ideas" (FERREE 2003, p. 308), los marcos pueden transformar el significado o la importancia de eventos o incidentes y así guiar la acción social (BENFORD; SNOW, 2000, p. 614). Al construir marcos, los actores de movimientos sociales pueden diagnosticar problemas sociales al identificar los factores $\mathrm{O}$ partidos responsables (BENFORD; SNOW, 2000). El enmarcamiento implica entonces un trabajo discursivo que, no obstante, puede ser disputado dentro de los mismos movimientos o bien sus opositores y los medios (BENFORD; SNOW, 2000, p. 623; FERREE, 2003). Este proceso no ocurre en un vacío: el entorno cultural y también las oportunidades políticas y discursivas afectan la articulación de marcos y sus resultados (FERREE, 2003; MCCAMMON et al., 2001; MCCAMMON et al., 2007; PEDRIANA, 2006).

Mi análisis está basado en 12 reportes elaborados ya sea de manera independiente o conjunta por NWAC y FAFIA como parte de su activismo 
doméstico y transnacional para reclamar que el estado canadiense se responsabilice por su falta de acción con respecto a MMIWG (Tabla 1). Yo elaboré todas las traducciones que aquí presento. Me enfoco en NWAC y FAFIA no sólo porque su colaboración refleja una importante alianza entre mujeres indígenas y feministas, sino también porque a través de ella lograron visibilizar nacional e internacionalmente la urgencia del problema de MMIWG. Esto no significa que no haya otras organizaciones indígenas de la sociedad civil que hayan hecho acciones similares.

Tabla 1. Reportes de NWAC y FAFIA, 2003-2016

\begin{tabular}{|c|c|c|c|}
\hline Año & FAFIA & NWAC & NWAC \& FAFIA \\
\hline 2003 & $\begin{array}{l}\text { "Canada's Failure } \\
\text { to Act: Inequality } \\
\text { Deepens," Report to } \\
\text { CEDAW }\end{array}$ & & \\
\hline 2007 & Report to CERD & & \\
\hline 2008 & $\begin{array}{l}\text { "Women's } \\
\text { Inequality in Canada," } \\
\text { Report to CEDAW }\end{array}$ & & \\
\hline 2009 & $\begin{array}{rrr}\text { "No } & \text { Action, } & \text { No } \\
\text { Progress," } & \text { Report } & \text { to } \\
\text { CEDAW } & & \end{array}$ & $\begin{array}{l}\text { "Voices of Our } \\
\text { Sisters In Spirit. Report } \\
\text { to Families and } \\
\text { Communities, 2 } \text { nd ed." }\end{array}$ & \\
\hline 2010 & & $\begin{array}{l}\text { "What Their } \\
\text { Stories Tell Us: } \\
\text { Research Findings from } \\
\text { the SIS Initiative." }\end{array}$ & \\
\hline
\end{tabular}




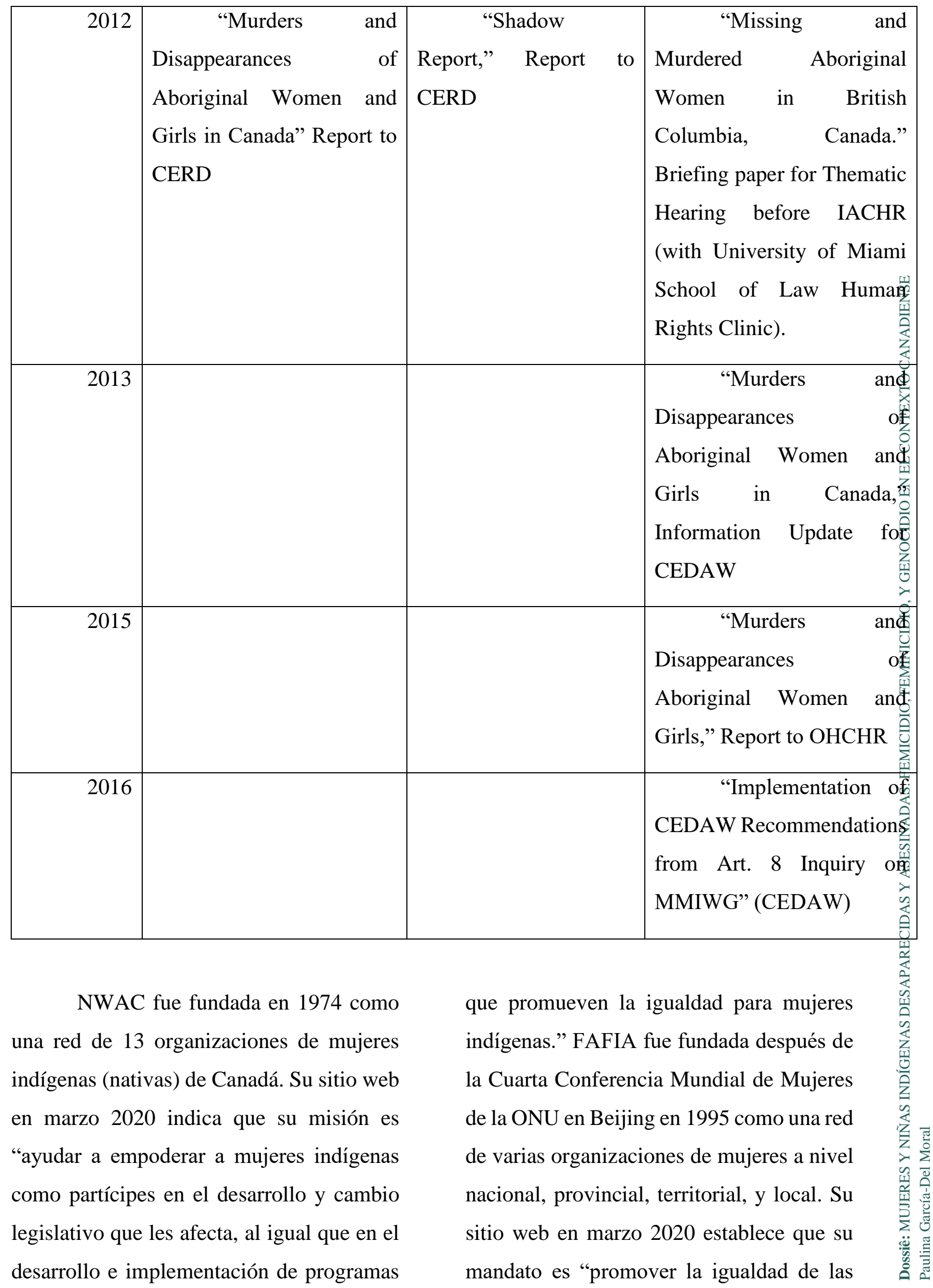


mujeres en Canadá trabajando hacia la implementación completa de los tratados y acuerdos internacionales que Canadá ha ratificado." Obtuve estos documentos de sus sitios web en el 2018. Algunos reportes son parte de la iniciativa "Hermanas en Espíritu" (Sisters in Spirit, SIS) de NWAC (2005-2010), la cual documentó el predominio de la violencia contra mujeres y niñas indígenas a pesar de la falta de datos confiables del gobierno canadiense. Los otros documentos que fueron presentados ante el Comité CEDAW, el Comité para la Eliminación de la Discriminación Racial (CERD), el Comité de Derechos Humanos (OHCHR, por sus siglas en inglés), $\mathrm{y}$ también la CIDH como parte de la colaboración entre NWAC y FAFIA en la "Campaña de Solidaridad con Mujeres Indígenas.” Esta campaña tuvo como objetivo que el Comité CEDAW y la CIDH condujeran visitas en Canadá para investigar MMIWG. Cabe notar aquí que estaba fuera del límite de este artículo analizar las respuestas del gobierno canadiense, los órganos de la ONU, y la

\footnotetext{
${ }^{5}$ Mi conceptualización de la violencia colonial y de la discriminación interseccional está basada en la literatura feminista indígena y antirracista ya revisada en la sección anterior. Esta perspectiva interseccional rechaza ver al género o a la raza como categorías primarias de análisis. Al contrario, adopta un marco "integrativo" para estudiar cómo el género y la raza se constituyen mutuamente de manera relacional. Esto significa que no podemos necesariamente hablar de la opresión de mujeres racializadas por su condición de género y raza, por el simple hecho de que todas y todos somos productos del género y la raza como estructuras sociales.
}

CIDH a estos marcos, aunque algunas están documentadas en los reportes que examiné.

Realicé un análisis cualitativo de los marcos que NWAC y FAFIA emplearon para problematizar y politizar MMIWG y tracé simultáneamente las oportunidades discursivas y políticas de las que dispusieron (FERREE, 2003; MCCAMMON et al., 2001; MCCAMMON et al., 2007). Generé los códigos de manera inductiva usando NVIVO en una primera lectura, y luego afiné mis criterios para codificar los marcos en una segunda lectura. Detecté dos marcos dominantes que están interrelacionados: uno construye el problema de MMIWG como violencia colonial que conlleva discriminación interseccional, y otro que la construye como una violación de derechos humanos 5 . Interesantemente, ningún reporte usa el término de genocidio. Los términos de feminicidio y femicidio tampoco están presentes.

\section{Enmarcando MMIWG: Violencia Colonial,}

De hacerlo, supondríamos que la raza no afecta a la gente blanca, o que el género afecta solamente a las mujeres. En otras palabras, volvemos invisibles a ciertas categorías de personas. Por lo tanto, es necesario prestarles atención a las condiciones materiales, a los procesos estructurales, así como a las representaciones culturales a través de los cuáles se construyen la raza y el género mutuamente y de manera relacional con el poder como un elemento constitutivo, a la vez tomando en consideración cómo la colonia es un factor integral en estos procesos. 
Discriminación Interseccional, y Derechos Humanos

NWAC y FAFIA generaron sus reportes en un contexto de activismo transnacional aumentado en respuesta a la resistencia del anterior Primer Ministro Harper a tomar seriamente el problema de MMIWG (KUBIK; BOURASSA, 2016; NAGY, 2016). En este sentido, los reportes representan "esfuerzos activos de enmarcamiento" (FERREE, 2003) para politizar la violencia contra las mujeres y niñas indígenas, así como para responsabilizar al Estado por su deficiente respuesta a ella. Como parte de esta estrategia, NWAC y FAFIA enmarcaron esta violencia como el producto de discriminación interseccional en contra de mujeres y niñas indígenas que emana del impacto histórico y presente del colonialismo y también como una violación de derechos humanos. El marco de violencia colonial y discriminación interseccional incluye referencias a la mayor vulnerabilidad a la que las mujeres y niñas indígenas se enfrentan. Específicamente, este marco construye una interpretación de esta vulnerabilidad como la consecuencia de la intersección de varias

\footnotetext{
${ }^{6}$ Cabe mencionar que NWAC y FAFIA se apoyan en un universo discursivo formado por otras organizaciones de la sociedad civil, como Amnistía Internacional, que usaron el marco de la discriminación para demostrar que
}

formas discriminación relacionadas a la pobreza, la raza, y el género en el contexto de la historia colonial de Canadá. El marco de la violación de derechos humanos incluye interpretaciones de esta discriminación sistémica como una muestra del incumplimiento de las obligaciones internacionales de Canadá bajo distintos tratados y acuerdos internacionales de derechos humanos para combatir la discriminación racial y de género, al igual que para prevenir, investigar, y castigar efectivamente la violencia contra las mujeres. ${ }^{6}$ También invoca el lenguaje de fallas del estado y de la impunidad, en especial cuando hay referencias a las semejanzas entre la inacción de México y de Canadá para responder a la violencia contra mujeres marginadas. En última instancia, los marcos se refuerzan, ya que ambos pintan un diagnóstico en donde la respuesta fallida del estado canadiense hacia la violencia contra mujeres y niñas indígenas y las condiciones históricas coloniales y estructurales fomentan el problema.

MMIWG como Violencia Colonial y

\section{Discriminación Interseccional}

El marco de la violencia colonial y la discriminación interseccional ligan la pobreza sistémica, el racismo, y el sexismo

Canadá no había actuado con la debida diligencia para prevenir, investigar, y sancionar las desapariciones y los asesinatos de mujeres y niñas indígenas. 
a los efectos históricos y concurrentes de la colonización. Este marco constituye la principal narrativa para interpretar el problema de MMIWG en todos los reportes

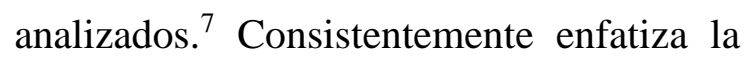
marginación de las mujeres y niñas indígenas y su relación con la violencia que se ejerce en su contra, así como la indiferencia por parte de las autoridades y la sociedad canadienses. El siguiente excepto es representativo de esta narrativa:

Las mujeres y niñas indígenas son uno de los grupos más desventajados social y económicamente en Canadá; muchas de esas desventajas están enraizadas en los efectos históricos y modernos de la colonización. [...] La violencia contra mujeres y niñas indígenas es la punta de un enorme iceberg de discriminación - relacionada al género, la clase, la raza, y el impacto de la colonización. [...] Esta compleja combinación ... puede explicar por qué y cómo es que tantas mujeres y niñas indígenas han desaparecido o sido asesinadas, al igual que la tan poca atención que se le ha dado a esta tragedia (NWAC; FAFIA; UM, 2012, p. $10)$.

NWAC y FAFIA también usan este marco para ligar la marginación de las mujeres y niñas indígenas a su vulnerabilidad a ser violentadas. NWAC

\footnotetext{
${ }^{7}$ Aunque no entro en este punto en mi análisis, quiero hacer notar que muchos de los documentos de FAFIA no se enfocan exclusivamente en MMIWG. De hecho, estos reportes son parte de los esfuerzos de FAFIA de abogar por los derechos de todas las mujeres en Canadá. El
}

(2009, p. 100; 2010, p. 7) argumenta que “el estrés socioeconómico" y el "racismo y patriarcado sistémicos" constituyen "un sistema de colonización" que crea "un clima en donde las mujeres indígenas son particularmente vulnerables a ser violentadas y victimizadas con indiferencia por parte del Estado y la sociedad a estas experiencias de violencia." Para FAFIA (2009, p. 19), el racismo y el sexismo son "raíces de las condiciones de marginación social y económicas de las mujeres indígenas" y que también "afectan las actitudes de los hombres violentos que las ven como blancos porque no cuentan con ninguna protección social dada la profundidad de la discriminación en su contra." Consecuentemente, "las mujeres y niñas indígenas encaran discriminación en múltiples frentes: como mujeres en sus comunidades de origen debido al legado del patriarcado impuesto durante la colonización, como mujeres en la sociedad general, y como personas indígenas en la sociedad general" (NWAC; FAFIA; UM, 2012, p. 18; NWAC; FAFIA, 2013).

\section{Además, NWAC y FAFIA} demuestran cómo las manifestaciones tanto públicas como privadas de esta discriminación interseccional son

marco de la violencia colonial y discriminación interseccional coexiste con un marco general de la violencia contra las mujeres como el producto de "la subordinación social, política, legal, y económica" y de la desigualdad sistémica (FAFIA, 2003, parr.176). 
legitimadas y reforzadas a través del derecho, "representando así desigualdades relacionadas con la falta de acceso a derechos sociales, económicos, culturales, políticos y civiles" (NWAC, 2012, p. 2; NWAC; FAFIA; UM 2012, p. 6; NWAC; FAFIA 2013, p. 16). Por ejemplo, FAFIA (2007, p. 21) toma la posición de que "el Indian Act no provee protecciones para las mujeres que son víctimas de abuso conyugal, a pesar de que las mujeres indígenas son particularmente vulnerables a ser sujetas de este tipo de abuso." El Indian Act y la sección 67 del Acta Canadiense de Derechos Humanos, que no permite que las mujeres indígenas interpongan demandas de discriminación de género en contra de Consejos de Bandas indígenas y hacer reclamos a su propiedad en reservas, exacerban esta vulnerabilidad y resultan en pobreza sistémica (FAFIA, 2003, parr. 16). La pobreza sistémica es mucho más seria para las trabajadoras sexuales indígenas: "La prostitución de mujeres y niñas indígenas es un reflejo de su situación económica y su marginación social. La prostitución incrementa su vulnerabilidad a ser coaccionadas, abusadas, y a ser víctima de violencia" (NWAC, FAFIA; UM 2012, p. 19).

También es importante notar que NWAC (2010, p. 2) asocia esta vulnerabilidad con la deshumanización de las mujeres y niñas indígenas como uno de los objetivos de la colonización: "La sobrerrepresentación de mujeres y niñas indígenas como víctimas de violencia en Canadá tiene que ser entendida en el contexto de una estrategia colonial que buscó deshumanizar a las mujeres indígenas." NWAC (2010, p. 33) también sitúa a los agresores indígenas de estas mujeres y niñas: "Aunque la historia no es una excusa para el comportamiento violento, la violencia ejercida por hombres indígenas se debe de considerar dentro de un contexto colonial que fomentó su devaluación como hombres, así como los sentimientos de impotencia que emanaron de esta situación." Sobre este trasfondo, FAFIA y NWAC argumentan que "la marginación social y económica de las mujeres y niñas indígenas las vuelve blancos fáciles para hombres violentos y a la vez es usada como justificación por parte de las autoridades para no protegerlas" (FAFIA, 2009; NWAC; FAFIA; UM, 2012, p. 4; NWAC; FAFIA, 2013).

En general, el enmarcamiento de la violencia contra mujeres y niñas indígenas como violencia colonial y discriminación interseccional produce una narrativa que politiza su gran vulnerabilidad a la violencia, su marginación, y la devaluación histórica de sus vidas. Este enfoque hace que NWAC y FAFIA sean críticas de 
algunas de las propuestas de prevención de violencia contra mujeres y niñas indígenas que "sitúan la responsabilidad en la víctima de velar por su seguridad" (NWAC, 2010, p. 32). En resumen, este marco apunta al Estado canadiense como el responsable de la violencia en contra de las mujeres y niñas canadienses, ya que liga la violencia colonial con la intersección de desigualdades sistémicas y su normalización a través del derecho o bien la falta de acceso de las mujeres y niñas a ejercer a derechos. El marco de la violación de derechos humanos está informado por este primer marco.

MMIWG como una Violación de

\section{Derechos Humanos}

NWAC y FAFIA utilizan el marco de la violación de derechos humanos principalmente para llamar atención a la negativa de la administración del Primer Ministro Stephen Harper de reconocer MMIWG como una "crisis" (NWAC; FAFIA, 2013, p. 39). La resistencia de su gobierno, de acuerdo con estas organizaciones, implica el incumplimiento de dos de sus obligaciones internacionales: 1) el ejercicio de la debida diligencia para prevenir, investigar, y remediar la violencia y 2) asegurarse de que las mujeres y niñas indígenas gocen y ejerzan el derecho a la vida, a la seguridad personal, a la igualdad bajo la ley, y a la igualdad de condiciones sociales y económicas (NWAC; FAFIA, 2013, p. 23).

El marco de la violación de derechos humanos crea una interpretación de la respuesta fallida del estado canadiense ante la crisis de MMIWG apoyándose en el marco de la violencia colonial y a la discriminación interseccional que analicé anteriormente: "Dado a que viven en el punto de intersección entre la discriminación de raza y de género, las mujeres indígenas se ven severamente impactadas por el legado de la colonia, el trato discriminatorio que reciben por parte de las instituciones y por la falta de protección a su persona cuando no se investiga la violencia ejercida en su contra" (NWAC; FAFIA; UM, 2012, p. 22). NWAC y FAFIA llaman la atención al fracaso del sistema de justicia de la Columbia Británica en el caso del asesino serial Robert Pickton para ilustrar este punto. Este fracaso reveló "las actitudes racistas y misóginas subyacentes en la cultura institucional de la policía y del Estado" (NWAC; FAFIA, 2013, p. 21). En efecto, para NWAC (2012, p. 8), "la policía canadiense y servidores públicos han hecho poco para prevenir el patrón de violencia racista hacia las mujeres indígenas, en parte porque ellos tienen actitudes racistas $\mathrm{y}$ discriminan así a las mujeres indígenas rutinariamente." 
En este contexto, la falta de debida diligencia "resulta en la impunidad de los crímenes y permite que la violencia continúe" (NWAC; FAFIA; UM, 2012, p. 5; NWAC; FAFIA, 2013). Al enmarcar MMIWG como una violación de derechos humanos, NWAC y FAFIA argumentan entonces que esta violencia resulta de fallas del Estado en dos niveles:

- la policía no protege a las mujeres y niñas indígenas de la violencia y tampoco investiga rápidamente casos de desapariciones o asesinatos;

- Canadá no ha remediado las condiciones de desventaja social y económica en las que las mujeres y niñas indígenas viven y que las hace vulnerables a la violencia o no les permite escaparla (NWAC; FAFIA; UM, 2012, p. 6; NWAC; FAFIA, 2013, p. 19).

Con base en este marco, NWAC y FAFIA invocan aquí los asesinatos de mujeres en Ciudad Juárez para requerir la intervención del Comité CEDAW y de la $\mathrm{CIDH}$, y así dar pie a la creación de un comité nacional de investigación sobre MMIWG en Canadá:

El Comité CEDAW ha ayudado tanto a las mujeres en México como al Estado mexicano a nivel nacional, estatal, y local en sus esfuerzos para responder a los asesinatos y las desapariciones de mujeres en Juárez. Nosotras queremos que las mujeres y niñas indígenas, así como Canadá, se beneficien de la experticia y la asistencia del Comité CEDAW en este momento para poder desarrollar métodos para hacer cumplir los derechos de las mujeres y niñas indígenas y para que Canadá cumpla con sus obligaciones de protegerlas de la violencia y de darles trato igualitario bajo la ley (NWAC; FAFIA, 2013, p. 38; énfasis de la autora).

A la fecha, la [Comisión] no ha examinado los asesinatos y las desapariciones sistémicas de las mujeres y niñas indígenas en Columbia Británica o en Canadá. Esta situación de crisis es paralela al caso de Campo Algodonero litigado en la Corte Interamericana de Derechos Humanos con respecto a los asesinatos de mujeres en Juárez. Una audiencia temática sería un método efectivo para llamar la atención regional e internacionalmente a este problema crítico de derechos humanos en Canadá.... Las peticionarias esperamos que la audiencia empuje al Gobierno de Canadá a comprometerse con las mujeres indígenas y las organizaciones que las representan para establecer un comité nacional de investigación sobre mujeres y niñas indígenas desaparecidas $y$ asesinadas.... (NWAC; FAFIA; UM, 2012, p. 27; énfasis de la autora).

El uso del marco de la violación de los derechos humanos por parte de NWAC y FAFIA claramente tiene como objetivo combatir la omisión e inacción del Estado en los casos de MMIWG al igual que los esfuerzos de Harper por despolitizar el problema. En este sentido, los esfuerzos activos de enmarcamiento de NWAC y FAFIA representan un ejercicio discursivo 
que busca responsabilizar al estado por ser cómplice en MMIWG y su impunidad.

\section{Discusión}

Aunque en este trasfondo NWAC y FAFIA hubieran posiblemente usado los términos de feminicidio o femicidio como marco en sus reportes, no es necesariamente sorprendente que no lo hicieran en el contexto canadiense. Las feministas indígenas simplemente no usan estos conceptos en su trabajo académico sobre MMIWG. El feminicidio, traducido como "feminicide" casi no es conocido, mientras que "femicide" sigue privilegiando principalmente al género como eje analítico por sobre de otros factores que contribuyen a MMIWG, como la colonización.

Tampoco sorprende que NWAC y FAFIA no hayan utilizado el concepto de genocidio como marco, a pesar de que las feministas indígenas lo emplean ampliamente. Los documentos de NWAC y FAFIA son reportes que fueron presentados a los órganos de la ONU que monitorean la implementación de la CEDAW, el CERD, o el Pacto Internacional de Derechos Civiles y Políticos por el Comité de Derechos Humanos. Estos órganos no monitorean la Convención para la Prevención y Sanción del Crimen de Genocidio. Por otra parte, algunos de los reportes tenían como propósito demandar una audiencia temática ante la CIDH y la intervención tanto de ésta como del Comité CEDAW a través de una visita al país. En otras palabras, NWAC y FAFIA se apoyaron en las oportunidades políticas y discursivas que les ofrecían los términos legales reconocidos por estos órganos internacionales y los tratados que monitorean, principalmente el lenguaje de la discriminación racial y de género, y de la debida diligencia para prevenir, investigar, y sancionar la violencia contra las mujeres, como lo estipulan la Recomendación \#19 de la CEDAW, la Convención Interamericana para Prevenir, Sancionar, y Erradicar la Violencia contra las Mujeres (Convención Belém Do Pará), y la Declaración Americana de los Derechos y Deberes del Hombre de la Organización de Estados Americanos (OEA). Si bien Canadá no ha firmado la Convención Belém Do Pará, la $\mathrm{CIDH}$ ha declarado previamente en el caso de Gonzáles Lenahan v. Estados Unidos que la obligación de los Estados de responder con medidas diligentes para prevenir la violencia contra las mujeres constituye derecho consuetudinario.

En resumen, el éxito de las estrategias de enmarcamiento de NWAC y FAFIA se refleja en las visitas e investigaciones que hicieron la CIDH y el Comité CEDAW (NAGY, 2016). Estas visitas, al igual que la audiencia temática ante la $\mathrm{CIDH}$, contribuyeron en parte a mermar la negativa atrincherada del Estado 
canadiense hacia reconocer la gravedad del problema de MMIWG y así dar pie eventualmente a la creación del Comité Nacional de Investigación cuando tuvo lugar el cambio de gobierno con Justin Trudeau como Primer Ministro.

\section{Conclusión}

Queda claro que para las académicas $\mathrm{y}$ activistas indígenas es de suma importancia conceptualizar MMIWG como genocidio. El nombrar así esta violencia ha sido elemental tanto para sanar como para resistir los esfuerzos del estado canadiense por despolitizar y permanecer indiferente ante el grave problema de MMIWG (JACOBS，2018; LAVELL-HARVARD; BRANT, 2016). Aunque NWAC y FAFIA no utilizan el concepto de genocidio, el enmarcamiento de MMIWG como violencia colonial y discriminación interseccional o como una violación de derechos humanos hace eco con la interpretación de este problema que propone el uso de genocidio.

\section{Esta interpretación de MMIWG} difiere de la definición del concepto de femicidio (femicide) que domina en el contexto canadiense, sobretodo en tanto que esta privilegia al género como eje analítico principal por sobre de la violencia colonial o de una perspectiva interseccional. En cambio, sí existen semejanzas entre la interpretación de MMIWG como genocidio y las definiciones latinoamericanas de feminicidio que enfatizan la devaluación de la vida de las mujeres y la complicidad del Estado en la violencia de género. Ambos conceptos ven al Estado como el principal actor de la violencia contra las mujeres. No obstante, como concepto, el feminicidio no toma en cuenta la violencia colonial ni racial, si bien sí enfatiza la marginación social y la pobreza (MONÁRREZ, 2002). Entonces también hay importantes diferencias entre la interpretación de MMIWG que produce el concepto de genocidio en una última instancia. De esto se desprenden preguntas con respecto a si el concepto de feminicidio en Latinoamérica puede capturar conceptualmente o atender de manera adecuada las experiencias de violencia de las mujeres y niñas indígenas o el impacto que ha tenido en ella el legado de racismo y pobreza de la colonia.

Los marcos de los movimientos sociales tienes implicaciones importantes. Como lo demuestra mi análisis, las estrategias discursivas de enmarcamiento de NWAC y FAFIA se basaron exitosamente en el lenguaje del derecho internacional para mermar la resistencia del estado a reconocer MMIWG y a crear un comité nacional que investigara este problema. Aunque no estudié la forma en 
que el Comité Nacional de Investigación sobre MMIWG utilizó el concepto de genocidio en su informe final para enmarcar esta violencia, fue muy importante que lo haya hecho; fue un shock para la sociedad canadiense. Sin embargo, sus 231 recomendaciones o "llamadas a la justicia" no han sido implementadas todavía. En Latinoamérica, en cambio, el uso de los términos feminicidio o femicidio han resultado en leyes innovadoras, si bien hay resistencia a su implementación. A pesar de lo cual un nuevo desarrollo es que la Comisión Interamericana de Mujeres ha propuesto una Ley Modelo para Prevenir, Sancionar y Erradicar la Muerte Violenta de Mujeres y Niñas (Femicidio/Feminicidio), posiblemente abriendo nuevas oportunidades para activistas feministas y de derechos humanos para demandar que los Estados latinoamericanos cumplan con su obligación de garantizar el derecho de las mujeres y niñas a una vida libre de violencia. Además, tanto la ONU y como la OEA han reconocido el femicidio y el feminicidio como problemas graves a través de declaraciones y la creación de protocolos para la investigación de estos crímenes. Puesto de manera diferente, los marcos de femicidio y feminicidio en Latinoamérica han tenido éxito transnacional. Pero precisamente por eso es importante tomar en cuenta las posibles limitaciones de estos términos y marcos, sobretodo en cuanto a su capacidad para conceptualizar la violencia contra mujeres y niñas indígenas. Si estos términos y marcos ignoran las experiencias de estas mujeres y niñas, su éxito institucional puede profundizar su exclusión social y política. También vale la pena preguntarse si la falta de una perspectiva antirracista o descolonial/anticolonial no ha permitido que las mujeres indígenas de Canadá se acerquen al movimiento feminista latinoamericano para combatir el femicidio/feminicidio. Aunque no puedo contestar esta pregunta, me queda claro que debe de haber un diálogo transnacional respetuoso y abierto entre las feministas y las mujeres indígenas de las Américas sobre la relación entre las violencias extremas contra las mujeres indígenas o racializadas y el colonialismo como una estructura de organización social que persiste en nuestro presente (véase FIGUEROA ROMERO et al., 2017). Espero que este artículo sea un paso en esa dirección.

\section{Referências bibliográficas}

ACOOSE, Janice. Iskewak-Kah 'Ki Yaw Ni Wahkomakanak: Neither Indian princesses nor easy squaws. Toronto: Women's Press, 1995.

AMNESTIIA INTERNACIONAL. "Stolen sisters. A human rights response to discrimination and violence against Indigenous women in Canada." 2004. 
https://www.amnesty.ca/sites/default/files/ amr200032004enstolensisters.pdf

ARVIN, Maile; TUCK, Eve; MORRILL, Angie. "Decolonizing feminism:

Challenging connections between settler colonialism and heteropatriarchy."

Feminist Formations, v. 25, n. 1, pp. 8-34, 2013.

ANDERSON, Brenda. "How the lens of the global locates Canada's missing and murdered Indigenous women: Pedagogical methods, lessons, and hope from the classroom." In: LAVELL-HARVARD, D. Memee; BRANT, Jennifer. Forever loved: exposing the hidden crisis of missing and murdered Indigenous women and girls in Canada, Canada: Demeter Press, 2016, pp. 194-211.

ANDERSON, Brenda; KUBIK, Wendee; HAMPTON, Mary Rucklos. Torn from our midst: Voices of grief, healing, and action from the Missing Indigenous Women Conference, 2008. Regina, Saskatchewan: University of Regina Press, 2010.

ANDERSON, Kim; CAMPBELL, Maria; BELCOURT, Christi. Keetsahnak/ Our missing and murdered Indigenous sisters. Kindle edition. Edmonton, Alberta: University of Alberta Press, 2018.

BENFORD, Robert D., SNOW, David A. "Framing processes and social movements: An overview and assessment." Annual Review of Sociology v. 26, pp. 611-639, 2000.

BOURGEOIS, Robyn. "Generations of genocide. The historical and sociological context of missing and murdered Indigenous women and girls." In: ANDERSON, Kim; CAMPBELL, Maria; BELCOURT, Christi. Keetsahnak/Our missing and murdered Indigenous sisters. Kindle edition. Edmonton, Alberta: University of Alberta Press, 2018.
BOURGEOIS, Robyn. "Perpetual state of violence: An Indigenous feminist antioppression inquiry into missing and murdered Indigenous women and girls." In: GREEN, Joyce. Making space for Indigenous feminism, 2a. ed. Canada: Fernwood Publishing, 2017, pp. 253-273.

BOURGEOIS, Robyn. "Colonial exploitation: The Canadian state and the trafficking of Indigenous women and girls in Canada." UCLA Law Review, v. 62, pp. 4126-4163, 2015.

BOYCE, Jillian. Victimization of Aboriginal people in Canada, 2014. Ottawa: Canadian Centre for Justice Statistics.

BRZOZOWKSI, Jodi-Anne; TAYLORBUTTS, Andrea; JOHNSON, Sara. "Victimization and offending among the Aboriginal population in Canada." Ottawa: Canadian Centre for Justice Statistics, 2006.

BULLER, Marion et al. Reclaiming power and place: The final report of the National Inquiry into missing and murdered Indigenous women and girls. Volume 1a, 2010. https://www.mmiwg-ffada.ca/finalreport/

CARTER, Sarah. Capturing Women: The manipulation of cultural imagery in Canada's Prairie West. Montreal: McGillQueen's University Press, 1997.

CFOJA. "Murdered and missing Indigenous women and girls." 2020. https://www.femicideincanada.ca/about/his tory/indigenous

CEDAW. "Report of the Inquiry Concerning Canada of the Committee on the Elimination of Discrimination against Women under Article 8 of the Optional Protocol to the CEDAW." CEDAW/C/OP.8/CAN/1. 2015. http://tbinternet.ohchr.org/Treaties/CEDA W/Shared\%20Documents/CAN/CEDAW_ 
C_OP-8_CAN_1_7643_E.pdf

CIDH. "Missing and murdered Indigenous women in British Columbia, Canada." 2014. OEA/Ser.L./V/II.Doc.30/14. https://www.oas.org/en/iachr/reports/pdfs/I ndigenous-Women-BC-Canada-en.pdf

CULHANE, Dara. "Their spirits live within us: Aboriginal women in Downtown Eastside Vancouver emerging into visibility." American Indian Quarterly, v. 27, n. 3\&4, pp. 593-606, 2003.

CONN, Heather. "Tina Fontaine." The Canadian Encyclopedia. 2019. https://www.thecanadianencyclopedia.ca/a rticle/tina-fontaine

CRAWFORD, Maria; GARTNER, Rosemary; DAWSON, Myrna. 1998. "Woman killing: Intimate femicide in Ontario, 1974-1994." Resources for Feminist Research, v. 26, n. 3-4.

DAWSON, Myrna. "106 Women and girls killed by violence: Eight-month report by the Canadian Femicide Observatory for Justice and Accountability." 2018. https://femicideincanada.ca/sites/default/fil es/2018-

09/CFOJA\%20FINAL\%20REPORT\%EN G\%20V3.pdf

DAWSON, Myrna. "Punishing femicide: Criminal justice responses to the killing of women over four decades." Current Sociology, v. 64, n. 7, pp. 996-1016, 2016.

DAWSON; Myrna; GARTNER,

Rosemary. "Differences in the characteristics of intimate femicides. The role of relationship state and relationship status." Homicide Studies, v. 2, n. 4, pp. 378-399, 1998.

DEER, Jessica. "Indian Act still discriminates against First Nations women, says UN Human Rights Committee." $C B C$ News, January 19, 2017. https://www.cbc.ca/news/indigenous/india n-act-sex-discrimination-un-committee1.4982330

DEPARTMENT OF JUSTICE CANADA. "Victimization of Indigenous women and girls." Just Facts, July 2017.

https://www.justice.gc/ca/eng/rp-pr/jr/jfpf/2017/docs/july05.pdf

EBERTS, Mary. "Being an Indigenous woman is a "high-risk lifestyle." In: GREEN, Joyce. Making space for Indigenous feminism, 2a. ed. Canada: Fernwood Publishing, 2017, pp. 69-102.

ERICKSON, Lesley. Westward bound: Sex, violence, the law, and the making of a settler society. Vancouver: University of British Columbia Press, 2011.

FAFIA. "Canada's failure to act: Women's inequality deepens. Submission of the FAFIA to the UN CEDAW Committee on the Occasion of Committee's Review of Canada's $5^{\text {th }}$ Report.” January 2003. http://www.fafia-afai.org/wpcontent/uploads/2015/02/1-2003-FAFIAReport-to-CEDAW.pdf

FAFIA. "Submission of the FAFIA to the UN CERD Committee on the Occasion of its Review of Canada's $17^{\text {th }}$ and $18^{\text {th }}$ Periodic Reports under the CERD." February 2007. http://www.fafiaafai.org/wp-content/uploads/2015/02/1FAFIA-Report-to-CERD-2007.pdf

FAFIA. "Women's inequality in Canada. Submission of the FAFIA to the UN CEDAW Committee on the Occasion of the Committee's Review of Canada's $6^{\text {th }} \&$ $7^{\text {th }}$ Period Reports." September 2008. http://www.fafia-afai.org/wpcontent/uploads/2015/02/3-2008-FAFIAReport-to-CEDAW.pdf

FAFIA. "No action, no progress. FAFIA report on Canada's progress in implementing priority recommendations made by the UN CEDAW Committee in 
2008." 2009.

http://www.cwhn.ca/sites/default/files/FAF IA\%20Statement.pdf

FAFIA. "Disappearances and murders of Aboriginal women and girls in Canada. Submission to the CERD Committee." January 2012. http://www.fafiaafai.org/wp-content/uploads/2015/02/3FAFIA-Report-to-CERD-2012.pdf

FAFIA and NWAC. "Reply to Issue 17. Implementation of CEDAW recommendations from Article 8 Inquiry on missing and murdered Indigenous women and girls." October 2017. http://fafia-afai.org/wpcontent/uploads/2016/10/Native-WomensAssociation-of-Canada-and-FAFIA.pdf

FEINSTEIN, Pippa; PEARCE, Megan. "Violence against Indigenous women and girls in Canada. Review of reports and recommendations - executive summary, 2015." Legal Strategy Coalition on Violence against Indigenous Women. https://www.leaf.ca/wpcontent/uploads/2015/02/ExecutiveSummary.pdf

FERREE, Myra Marx. "Beyond resonance and radicalism: Feminist framing of the abortion debates in the United States and Germany." American Journal of Sociology, v. 109, n. 2, pp. 304-344, 2003.

FIDAN, Caroline; DOENMEZ, Tyler. "The unmournable body of Cindy Gladue: On corporeal integrity and grievability." In: LAVELL-HARVARD, D. Memee; BRANT, Jennifer. Forever loved: exposing the hidden crisis of missing and murdered Indigenous women and girls in Canada, Canada: Demeter Press, 2016, pp. 111-127.

FIGUEROA ROMERO, Dolores et al.

"Virtual forum: Violence against Indigenous women in the Americas, February 6-March 10, 2017. Final summary report." 2017. Canadian Association of Latin American and
Caribbean Studies. http://www.canlatam.org.

FREGOSO, Rosa-Linda; BEJARANO, Cynthia. Terrorizing Women: Feminicide in the Americas. Durham, NC: Duke University Press, 2010.

GARCÍA-DEL MORAL, Paulina. "The murders of Indigenous women as feminicides: Toward a decolonial intersectional reconceptualization of femicide." Signs: Journal of Women in Culture and Society, v. 43, n. 4, pp. 929954, 2018.

GLENN, Evelyn Nakano. "Settler colonialism as a structure: A framework for comparative studies of US race and gender formation." Sociology of Race and Ethnicity, v. 1, n. 1, pp. 52-72, 2015.

GOULDING, Warren. Just another Indian: A serial killer and Canada's indifference. Calgary: Heritage House Publishing Company, 2001.

GREEN, Joyce. "Taking more account of Indigenous feminism: An introduction." In: GREEN, Joyce. Making space for Indigenous feminism, 2a. ed. Canada: Fernwood Publishing, 2017a, pp. 1-20.

HARGREAVES, Allison. Violence against Indigenous women. Waterloo: Wilfried Laurier University Press, 2017.

HUMAN RIGHTS WATCH. "Those who take us away: Abusive policing and failures in protection of Indigenous women and girls in Northern British Columbia, Canada." 2013.

https://www.hrw.org/report/2013/02/13/tho se-who-take-us-away/abusive-policingand-failures-protection-indigenous-women

INNES, Robert Alexander; ANDERSON Kim. "The moose in the room. Indigenous men and violence against women." In: ANDERSON, Brenda; KUBIK Wendee; HAMPTON Mary Rucklos. Torn from our 
midst: Voices of grief, healing, and action from the Missing Indigenous Women Conference, 2008. Regina, Saskatchewan: University of Regina Press, 2010.

JACOBS, Beverly. "Honouring women." In: ANDERSON, Kim; CAMPBELL, Maria; BELCOURT, Christi. Keetsahnak/ Our missing and murdered Indigenous sisters. Kindle edition. Edmonton, Alberta: University of Alberta Press, 2018.

JIWANI, Yasmin; YOUNG, Mary Lynn. "Missing and murdered women:

Reproducing

marginality in news discourse." Canadian Journal of Communication, v. 31, n. 4, pp. 895-917, 2006.

KOUKANNEN, Rauna. "Gendered violence and politics in Indigenous communities." International Feminist Journal of Politics, v. 17, n. 2, pp. 271288, 2014.

KNOTT, Helen. "Violence and extraction. Stories form the oil fields." In:

ANDERSON, Kim; CAMPBELL, Maria; BELCOURT, Christi. Keetsahnak/ Our missing and murdered Indigenous sisters. Kindle edition. Edmonton, Alberta: University of Alberta Press, 2018.

KUBIK, Wendee; BOURASSA, Carrie. "The politics, policies, and travesty of missing and murdered women in Canada." In: LAVELL-HARVARD, D. Memee; BRANT, Jennifer. Forever loved: exposing the hidden crisis of missing and murdered Indigenous women and girls in Canada, Canada: Demeter Press, 2016, pp. 17-33.

LAGARDE, Marcela. "Por la vida y libertad de las mujeres. Fin al feminicidio." Fem v. 28, n. 255, pp. 26-34, 2004.

LAVELL-HARVARD; D. Memee; BRANT, Jennifer. Forever loved: exposing the hidden crisis of missing and murdered Indigenous women and girls in Canada.
Canada: Demeter Press, 2016.

LINDBERG, Tracey; CAMPEAU, Priscilla; CAMPBELL, Maria. "Indigenous women and sexual assault in Canada." In: SHEEHY, Elizabeth, Sexual assault in Canada: Law, legal practice, and women's activism. Ottawa: University of Ottawa Press, 2012; pp. 87-110.

LSC. "Part of the solution, or part of the problem? The RCMP Update 2015. A Comment by the Legal Strategy Coalition on Violence against Indigenous Women on the RCMP update to the National Operational Review, 2015.” 2015. https://www.leaf.ca/wpcontent/uploads/2015/06/LSC-Review-of2015-RCMP-Update.pdf

LUCCHESI, Annita Hetoevéhotokke'e. "Mapping geographies of Canadian colonial occupation: Pathway analysis of murdered Indigenous women and girls." Gender, Place \& Culture, v. 26, n. 6, pp. 868-887, 2019.

\section{MANITOBA AVOCATE FOR}

CHILDREN AND YOUTH (MACY). "A

place where it feels like home: The story of Tina Fontaine.” 2019.

https://manitobaadvocate.ca/wpcontent/uploads/MACY-Special-ReportMarch-2019-Tina-Fontaine-FINAL1.pdf

MCCAMMON, Holly J. et al. "How movements win: Gendered oppportunity structures and U.S. women's suffrage movements, 1866-1919." American Sociological Review, v. 66, n. 1, pp. 49-70, 2001.

MCCAMMON, Holly J. et al. "Movement framing and discursive opportunity structures: The political successes of the U.S. women's jury movements." American Sociological Review, v. 72, n. 5, pp. 725749, 2007.

MENJÍVAR, Cecilia; WALSH, Shannon Drysdale. "The architecture of feminicide: 
The state, inequalities and everyday gender violence in Honduras." Latin American Research Review, v. 52, n. 2: 221-240, 2017.

MENJÍVAR, Cecilia; WALSH, Shannon Drysdale. "Subverting justice: Socio-legal determinants of impunity for violence against women in Guatemala. Laws, v. 5, n. 3, pp. 31-51, 2016.

MONÁRREZ, Julia. "Feminicidio sexual serial en Ciudad Juárez: 1993-2001.” Debate Feminista, v. 25, pp. 279-305.

MILLION, Dian. Therapeutic nations: Healing in the age of Indigenous human rights. Tucson: University of Arizona Press, 2011.

NAGY, Rosemary. "Transnational advocacy for the missing and murdered Indigenous women." In: LAVELLHARVARD, D. Memee; BRANT, Jennifer. Forever loved: exposing the hidden crisis of missing and murdered Indigenous women and girls in Canada, Canada: Demeter Press, 2016, pp. 177-193.

NWAC. 2018. Report card. The National Inquiry into Missing and Murdered Indigenous Women.

https://www.nwac.ca/wpcontent/uploads/2018/05/NWACMMIWG-Report-Card-3-May17Mar18.pdf

NWAC. 2017. Report card. The National Inquiry into Missing and Murdered Indigenous Women.

https://www.nwac.ca/wpcontent/uploads/2017/01/NWAC-InquiryReport-Card-and-Backgrounder-Jan2017.pdf

NWAC. More than invisible, invisible to real action. $N W A C$ 's response to the Special Committee on Violence against Indigenous Women (SCVAIW), 2014. https://cwpcsp.ca/resources/sites/default/files/resource
s/ENG_NWAC_Offical_response_to_SCV AIW.pdf

NWAC. 2010. "What their stories tell us: Research findings from the Sisters In Spirit initiative." https://www.nwac.ca/wpcontent/uploads/2015/07/2010-WhatTheir-Stories-Tell-Us-Research-FindingsSIS-Initiative.pdf

NWAC. 2009. Voices of our Sisters In Spirit. Report to families and communities, $2^{\text {nd }}$ ed. https://www.nwac.ca/wpcontent/uploads/2015/05/NWAC_Voicesof-Our-Sisters-In-Spirit_2nd-

Edition_March-2009.pdf

NWAC; FAFIA; UNIVERSITY OF MIAMI LEGAL CLINIC. Missing and Murdered Aboriginal Women and Girls in British Columbia, Canada. Briefing Paper for Thematic Hearing before the IACHR, 144th Period of Sessions, March 28, 2012, 2012. http://www.fafia-afai.org/wpcontent/uploads/2014/09/3-IACHRCanada-Briefing-Paper-March-282012.pdf

NWAC; FAFIA. Murders and Disappearances of Aboriginal Women and Girls in Canada. Information Update for the UN Committee on the Elimination of Discrimination against Women, 2013. http://www.fafia-afai.org/wpcontent/uploads/2014/09/5-

NWACFAFIAapplicationforhearing2013.p df

O'REILLY AND FLEMMING, “'Only the silence remains': Aboriginal women as victims in the case of the Lower Eastside (Pickton) murders. Investigative flaws, and the aftermath of violence in Vancouver." In: LAVELL-HARVARD, D. Memee; BRANT, Jennifer. Forever loved: exposing the hidden crisis of missing and murdered Indigenous women and girls in Canada, Canada: Demeter Press, 2016, pp. 47-78.

PALMATER, Pamela. "Shining light on the dark places: Addressing police racism 
and sexualized violence against Indigenous women and girls in the National Inquiry."

Canadian Journal of Women and the Law, v. 28, n. 2, pp. 253-284, 2016.

PEDRIANA, Nicholas. "From protective to equal treatment: Legal framing processes and

transformation of the women's movement in the 1960s." American Journal of Sociology v.111, n. 6,

pp. 1718-1761, 2006.

RAZACK, Sherene. "Gendering Disposability." Canadian Journal of Women and the Law, v. 28, n. 2, pp. 285307, 2016.

RAZACK, Sherene. "Gendered racial violence and spatialized justice: The murder of Pamela George." In: RAZACK, Sherene. Race, space, and the law: Unmapping a white settler society. Toronto: Between the Lines, 2002, pp. 121-155.

RADFORD, Jill; RUSSELL, Diana E.H. Femicide: The politics of woman-killing. New York: Twayne.

\section{ROYAL CANADIAN MOUNTED} POLICE (RCMP). 2014.

https://www.rcmp-grc.gc.ca/en/missingand-murdered-aboriginal-women-nationaloperational-overview

RCMP. Missing and murdered Aboriginal women. 2015 Update to the National Operational Overview, 2015.

https://www.recmpgrc.gc.ca/wam/media/455/original/c3561a2 84cfbb9c244ef57750941439.pdf

RIEL-JOHNS, Jessica. "Understanding violence against Indigenous women and girls in Canada." In: LAVELLHARVARD, D. Memee; BRANT, Jennifer. Forever loved: exposing the hidden crisis of missing and murdered
Indigenous women and girls in Canada, Canada: Demeter Press, 2016, pp. 34-46.

RUSSELL, Diana E.H.; HARMES, Roberta. Femicide in global perspective. New York and London: Teachers College, Columbia University.

SABO, Don. "Highway of tears." The Canadian Encyclopedia, 2016.

https://www.thecanadianencyclopedia.ca/e n/article/highway-of-tears

SAVARESE, Josephine L. “Analyzing erasures and resistance involving Indigenous women in New Brunswick, Canada." In: LAVELL-HARVARD, D. Memee; BRANT, Jennifer. Forever loved: exposing the hidden crisis of missing and murdered Indigenous women and girls in Canada, Canada: Demeter Press, 2016, pp. 128-159.

SMITH, Andrea. Conquest: Sexual violence and American Indian genocide. Durham: Duke University Press, 2005.

STASIULIS, Daiva; JHAPPAN, Radha. "The fractious politics of settler society: Canada." In: STASIULIS, Daiva; YUVAL-DAVIS, Nira. Unsettling settler societies: Articulations of gender, race, ethnicity and class. London: Sage, 1995, pp. 95-131.

STEVENSON, Winona. "Colonialism and First Nations women in Canada." In: DUA; Enashki; ROBERTSON, Angela.

Scratching the surface: Canadian antiracist feminist thought, Toronto, ON: Women's Press, 1999, pp. 49-82.

STATISTICS CANADA. Table 35-100156-01. Number, percentage, and rate of homicide victims, by sex and Aboriginal identity, 2020. DOI:

https://doi.org/10.25318/3510015601-eng.

SUZACK, Cheryl. Indigenous women and feminism: politics, activism, culture. 
Vancouver: University of British Columbia Press, 2010.

DAVIS, Nira. Unsettling settler societies: Articulations of gender, race, ethnicity and class. London: Sage, 1995, pp. 95-131.

STEVENSON, Winona. "Colonialism and First Nations women in Canada." In: DUA; Enashki; ROBERTSON, Angela.

Scratching the surface: Canadian antiracist feminist thought, Toronto, ON:

Women's Press, 1999, pp. 49-82.

STATISTICS CANADA. Table 35-100156-01. Number, percentage, and rate of homicide victims, by sex and Aboriginal identity, 2020. DOI:

https://doi.org/10.25318/3510015601-eng.

SUZACK, Cheryl. Indigenous women and feminism: politics, activism, culture.

Vancouver: University of British Columbia Press, 2010. 